\title{
Desarrollo de la Competencia Interpretativa desde las Clases de Química en Estudiantes de Ingeniería de la Universidad Santo Tomás de Aquino
}

\author{
Development of Interpretive Competition from \\ Chemistry Classes of Engineering Students from the \\ University of Santo Tomas de Aquino
}

Cristian Eduardo Cortés Aguilón ${ }^{1}$

Angie Paola Mahecha Vargas ${ }^{2}$

Maritza Angélica Murcia Hernández ${ }^{3}$

\section{Resumen}

El presente artículo evidencia los resultados obtenidos en el proyecto de investigación titulado Desarrollo de la Competencia Interpretativa desde las Clases de Química, a través de la aplicación y desarrollo de una secuencia didáctica en los temas, reacciones químicas, estequiometria y disoluciones, en el espacio académico de química general con estudiantes de ingeniería de primer semestre de la Universidad Santo de Tomás de Aquino. Estos estudiantes están adscritos a programas profesionales como ingeniería mecánica e ingeniería electrónica, sus edades oscilan entre 17 a 20 años. La metodología se desarrolló en tres fases, así: Fase preliminar (construcción y aplicación de una secuencia didáctica, la cual constó de tres secciones, iniciación, desarrollo y finalización); Fase de aplicación de instrumentos de recolección de información (se diseñaron y aplicaron tres instrumentos tipo Likert), y por último, la Fase de interpretación y análisis de resultados (fueron muy importantes las actividades de iniciación, las actividades de desarrollo y las actividades de finalización).

1 Profesor en formación Departamento de Química, Universidad Pedagógica Nacional. Correo electrónico: dqu_cecortesa468@pedagogica.edu.co

2 Profesor en formación Departamento de Química, Universidad Pedagógica Nacional. Correo electrónico: dqu_apmahechav575@pedagogica.edu.co.

3 Profesor en formación Departamento de Química, Universidad Pedagógica Nacional. Correo electrónico: dqu_mamurciah295@pedagogica.edu.co. 
Las actividades de la secuencia didáctica tuvieron como objetivo evaluar la competencia interpretativa dividida en tres dimensiones, las cuales son: resolutiva, lecto-escritora y gráfica. Con dicha secuencia didáctica se logró que el estudiante alcanzara una mayor comprensión de las temáticas desarrolladas en la asignatura de Química General, y la aplicabilidad de estos temas en la ingeniería; además, el desarrollo implícito de las habilidades interpretativas correspondientes a cada dimensión. Por tanto podemos afirmar que la importancia de la aplicación de una secuencia didáctica radica tanto en el planteamiento de las situaciones que sirven como pretexto para comprender y enfrentar la realidad, como en el desarrollo de habilidades que se deben poner en juego para que las actividades planeadas logren los objetivos que van acorde tanto con los intereses de los estudiantes y con los diferentes contenidos que permiten motivar y lograr mejores resultados a la hora de interpretar.

\section{Palabras clave}

Competencia interpretativa; Secuencia didáctica; dimensión resolutiva; habilidades, ingeniería.

\section{Abstract}

This research project demonstrates the application and development of a teaching sequence on issues, chemical reactions, stoichiometry and solutions in the academic space of general chemistry with engineering students from first semester at the Santo Tomas de Aquino University, which are assigned to professional programs such as mechanical engineering and electronic engineering, their ages range from 17 to 20 years. The methodology had three phases as follows: Preliminary phase: construction and application of a didactic sequence, which consisted of three sections, initiation, development and completion. Implementation phase of instruments for collecting information on which were designed and implemented three instruments Likert and finally, the phase of interpretation and analysis of results, which were very important initiation activities, development activities and activities ending. The activities of the teaching sequence aimed to assess the interpretative jurisdiction, its dimensions taking each a graphical approach, reading and writing and problem-solving. With this sequence, it was possible that the student attain a greater understanding of the themes developed in the course of general chemistry, and the applicability of these topics in engineering, also the implicit development of interpretive skills, such as graphics, reading-writing and problem-solving. Therefore we can say that the importance of the application of a teaching sequence lies both in the approach of the situations that serve as a pretext to understand and face reality, and the development of skills that must be put into play for the planned activities achieve the objectives that align the interests of both students and with different content that allow motivate and achieve better results when interpreting.

\section{Keywords}

Interpretative competence; teaching sequence; operative dimension; skills, engineering.

\section{Introducción}

El proyecto PEP (Proyecto Educativo del Programa) de ingeniería mecánica, de la Universidad Santo Tomás, busca la formación integral de los estudiantes, de manera que logren un desarrollo como seres humanos en sus múltiples dimensiones. Asimismo se busca que identifiquen problemas, desarrollen competencias y actúen éticamente. (USTA, 2015). De acuerdo a esto, se ve la necesidad de potenciar las habilidades y con ellas las competencias, en este caso la competencia interpretativa mediante el abordaje de una estrategia de secuencia didáctica con actividades que desarrollen en el estudiante la habilidad de interpretar diferentes situaciones y así resolver problemas de carácter científico.

Por lo anterior, la pregunta problema pretende partir de la creación y aplicación de una secuencia didáctica que pueda evidenciar si es posible potenciar en los estudiantes las habilidades correspondientes a la competencia interpretativa, pertenecientes a las dimensiones lecto-escritora, gráfica y resolutiva. 
Para ello, las actividades de iniciación, desarrollo y finalización que, al momento de ser aplicadas y analizadas, se fueron contrastando con los instrumentos diseñados, los cuales tuvieron como objetivo reconocer las herramientas interpretativas empleadas por los estudiantes, el nivel de dificultad que presentaban los diversos procesos de interpretación en temáticas de la química trabajados y la utilidad o pertinencia que representó para ellos la aplicación de la secuencia.

La formación para un estudiante debe dar herramientas para una vida social que va a exigir iniciativa y apertura, flexibilidad y criterio; se debe formar individuos capaces de formular sus propios problemas y de interpretar circunstancias inesperadas. Estas consideraciones justificarían la decisión de pensar la educación en términos de ampliar las posibilidades de acción, interacción e interpretación de los estudiantes y no solo de asegurar la adquisición de ciertos conocimientos. Lo que existe entre nosotros o en nuestro entorno tiene un significado para los seres humanos cuando es nombrado, cuando es interpretado; el mundo que habitamos, entonces, es inevitablemente un mundo interpretado. La competencia interpretativa es propia de todos los seres humanos (Toro, Reyes y Martínez, 2007).

Es por esto que en los procesos de formación profesional es de gran importancia, inicialmente, la adquisición y el posterior desarrollo de habilidades específicas que pueden llegar a ser potenciadas como competencia, las cuales son de gran ayuda en el desempeño en ámbitos laborales futuros. Desarrollar la competencia interpretativa en el aprendizaje de contenidos científicos en caso particular de la química es de vital importancia, ya que permite que el individuo pueda apropiar, explicar y describir de forma más significativa y específica el reconocimiento de los fenómenos.

Por lo anterior y acorde a lo expuesto por Martínez y Pinto (2009) en su trabajo, con el que busca articular una química aplicable a ingenierías industriales (mecánica, industrial y aeronáutica) ingeniería civil y ambiental, es esencial la fundamentación, comprensión e interpretación de los procesos que son base para el aprendizaje de la química.

\section{Marco de referencia}

La competencia interpretativa representa una oportunidad para desarrollar procesos comprensivos y transformar el aprendiz en sujeto lector motivado, involucrado y comprometido. En lugar de responder preguntas y "dar cuenta de" (entiéndase reproducir) lo leído, el estudiante tiene espacio para formular preguntas y cuestionar el discurso, el cual, como se ha mencionado, habrá de ser no solo verbal sino pictórico, icónico, audiovisual, hipertextual, es posible lograr de él una actitud más dispuesta y un trabajo más cooperativo (Vargas, 2008).

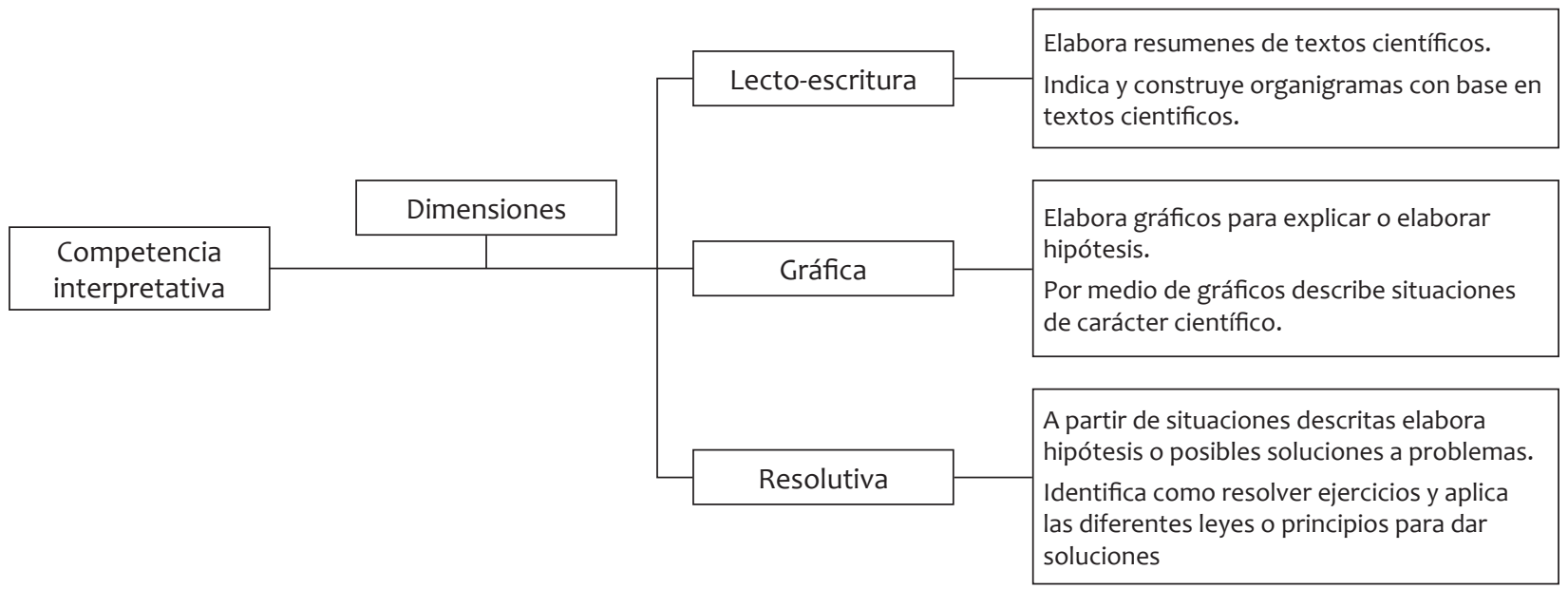

Gráfico 1 Funcionamiento de la competencia interpretativa, vista desde tres dimensiones, cada una con sus indicadores evaluables. 
Saber interpretar es una actividad sistemática y rigurosa, pues se trata de un proceso de reconstrucción y construcción de significados, formas de significar, de atribuir sentido y de actuar, en conformidad. Es decir, el estudiante, mediante tal actividad, ha de aproximarse paulatinamente a la manera de interpretar estructuras conceptuales y metodológicas. Se trata del cambio en los conceptos, en las metodologías, en la mirada estética, en las actitudes y en las valoraciones con las que los estudiantes ingresan para sumergirse creativamente en el lenguaje y procederes de las ciencias (Gallego, 2011).

\section{Metodología}

El proyecto se desarrolló en las siguientes fases:

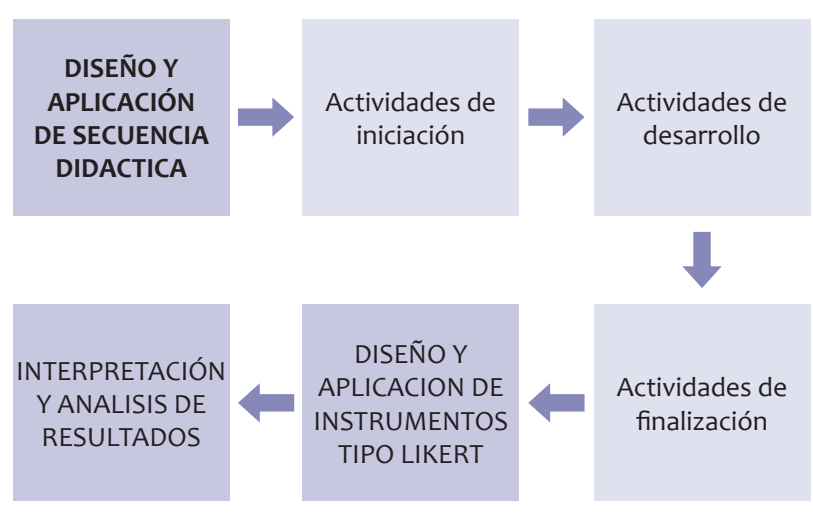

Gráfico 2 Descripción de la metodología

Fuente: Elaboración Propia

1. Fase preliminar: Construcción y aplicación de la secuencia didáctica, la cual constó de tres secciones (ver tabla 1): iniciación, desarrollo y finalización; estas están compuestas por actividades que abarcan temas de química como formulación y clasificación de reacciones químicas, reacciones de óxido reducción y balanceo redox, cálculos químicos, composición porcentual, fórmula empírica y fórmula molecular, relaciones molares y estequiometria.

\section{Fase de aplicación de instrumentos de recolec- ción de información:}

Diseño y aplicación de tres instrumentos tipo Likert, basados en los resultados obtenidos en la observación realizada en la práctica pedagógica y didáctica I (ver tabla 2):

Tabla 1. Instrumentos de recolección de información y actividades de secuencia didáctica

\begin{tabular}{|l|l|}
\hline $\begin{array}{l}\text { Actividad } \\
\text { instrumento }\end{array}$ & Concepto \\
\hline Actividades de iniciación \\
\hline Actividad 1 & Reconocimiento e interpretación de diagramas de procesos. \\
\hline Actividad 2 & Interpretación de textos científicos. \\
\hline Actividades de desarrollo \\
\hline Actividad 3 & $\begin{array}{l}\text { Interpretación de analogías y enunciados para la resolución de } \\
\text { problemas. }\end{array}$ \\
\hline Actividad 4 & $\begin{array}{l}\text { Interpretación de gráficos para la resolución de problemas sobre } \\
\text { reacciones químicas. }\end{array}$ \\
\hline Actividades de finalización \\
\hline Actividad 5 & $\begin{array}{l}\text { Interpretar reacciones químicas, realizar cálculos estequiométricos a } \\
\text { partir de gráficos. }\end{array}$ \\
\hline Actividad 6 & $\begin{array}{l}\text { Interpretación de textos y diagramas científicos, resolución de } \\
\text { problemas a partir de las leyes de los gases ideales }\end{array}$ \\
\hline Instrumentos de recolección de información \\
\hline Instrumento 1 & Medir la frecuencia del desarrollo de actividades interpretativas \\
\hline Instrumento 2 & $\begin{array}{l}\text { Evaluación de la competencia interpretativa de acuerdo al grado de } \\
\text { dificultad expresado por los estudiantes en el transcurso del desarrollo } \\
\text { de la secuencia didáctica. }\end{array}$ \\
\hline Instrumento 3 & $\begin{array}{l}\text { Evaluación final del desarrollo de la competencia interpretativa en los } \\
\text { estudiantes. }\end{array}$ \\
\hline
\end{tabular}

Tabla 1 Relación de instrumentos de recolección de información y actividades de secuencia didáctica.

Fuente Elaboración propia

3. Fase de interpretación y análisis de resultados: esta fase fue producto de tres fuentes de análisis, así: instrumentos de recolección de información, desarrollo de la secuencia didáctica y la observación realizada en el proceso de aplicación de la misma.

\section{Resultados}

El instrumento 1 (ver anexo 1) se dividió en cuatro categorías como se muestra en la siguiente tabla:

Tabla 2. Resultados de instrumento 1

Habilidades interpretativas
\begin{tabular}{|c|c|c|c|c|}
\hline Categoria & Item & $\begin{array}{c}\text { Siempre } \\
(\%)\end{array}$ & $\begin{array}{c}\text { Algunas } \\
\text { veces }(\%)\end{array}$ & Nunca $(\%)$ \\
\hline \multirow{3}{*}{ Lectora } & 2 & 0,0 & 42,9 & 57,1 \\
\cline { 2 - 5 } & 6 & 14,3 & 78,6 & 7,1 \\
\cline { 2 - 5 } & 7 & 71,4 & 21,4 & 7,1 \\
\hline \multirow{2}{*}{ Observacional } & 1 & 7,1 & 71,4 & 21,4 \\
\cline { 2 - 5 } & 8 & 14,3 & 78,6 & 7,1 \\
\hline Entorno & 3 & 35,7 & 64,3 & 0,0 \\
\cline { 2 - 5 } educativo & 10 & 0,0 & 35,7 & 64,3 \\
\hline \multirow{3}{*}{ Conceptual } & 4 & 14,3 & 64,3 & 21,4 \\
\cline { 2 - 5 } & 5 & 21,4 & 64,3 & 14,3 \\
\cline { 2 - 5 } & 9 & 64,3 & 35,7 & 0,0 \\
\hline
\end{tabular}

Tabla 2 Resultados instrumento 1 Fuente: Elaboración propia. 
Para el análisis del instrumento 2 (ver anexo 2) se realizó una matriz por cada dimensión, que permitió contrastar las actividades desarrolladas en la secuencia didáctica.
Para el instrumento 3 (ver anexo 3), el puntaje que los estudiantes le dieron a cada ítem se dividió en tres categorías, las cuales son: bajo (1.0-3.5), medio (3.6-4.4) y alto (4.5-5.0), expresando los resultados de este en porcentaje y evidenciados en la tabla 3.

Tabla 3. Resultados de instrumento 3

\begin{tabular}{|c|c|c|c|c|c|c|c|c|c|c|c|c|}
\hline \multirow{2}{*}{\begin{tabular}{|c} 
Estrategia \\
metodológica
\end{tabular}} & \multicolumn{3}{|c|}{ Recuerdo la actividad } & \multicolumn{3}{|c|}{$\begin{array}{l}\text { Utilidad para } \\
\text { asimilación de } \\
\text { conceptos }\end{array}$} & \multicolumn{3}{|c|}{$\begin{array}{l}\text { Utilidad para } \\
\text { favorecer el interés }\end{array}$} & \multicolumn{3}{|c|}{$\begin{array}{l}\text { Relación con la } \\
\text { ingeniería }\end{array}$} \\
\hline & Bajo & Medio & Alto & Bajo & Medio & Alto & Bajo & Medio & Alto & Bajo & Medio & Alto \\
\hline $\begin{array}{l}\text { 1. Reconocimiento } \\
\text { e interpretación de } \\
\text { diagramas de proceso }\end{array}$ & $33.3 \%$ & $33.3 \%$ & $33.3 \%$ & $41.7 \%$ & $25 \%$ & $33.3 \%$ & $41.7 \%$ & $33.3 \%$ & $25 \%$ & $41.7 \%$ & $25 \%$ & $33.3 \%$ \\
\hline $\begin{array}{l}\text { 2. Interpretación de } \\
\text { textos cientificos }\end{array}$ & $50 \%$ & $8.3 \%$ & $41.7 \%$ & $16.7 \%$ & $66.7 \%$ & $16.7 \%$ & $41.7 \%$ & $33.3 \%$ & $25 \%$ & $25 \%$ & $41.7 \%$ & $33.3 \%$ \\
\hline $\begin{array}{l}\text { 3. Interpretación } \\
\text { de analogías y } \\
\text { enunciados para } \\
\text { la resolución de } \\
\text { problemas. }\end{array}$ & $66.7 \%$ & $0 \%$ & $33.3 \%$ & $50 \%$ & $16.7 \%$ & $33.3 \%$ & $50 \%$ & $16.7 \%$ & $33.3 \%$ & $50 \%$ & $33.3 \%$ & $16.7 \%$ \\
\hline $\begin{array}{l}\text { 4. Interpretación } \\
\text { de gráficos para } \\
\text { la resolución de } \\
\text { problemas sobre } \\
\text { reacciones químicas. }\end{array}$ & $33.3 \%$ & $33.3 \%$ & $33.3 \%$ & $8.3 \%$ & $75 \%$ & $16.7 \%$ & $41.7 \%$ & $41.7 \%$ & $16.7 \%$ & $16.7 \%$ & $58.3 \%$ & $25 \%$ \\
\hline $\begin{array}{l}\text { 5. Interpretar } \\
\text { reacciones químicas, } \\
\text { realizar cálculos } \\
\text { estequiométricos a } \\
\text { partir de gráficos. }\end{array}$ & $41.7 \%$ & $33.3 \%$ & $25 \%$ & $41.7 \%$ & $33.3 \%$ & $25 \%$ & $41.7 \%$ & $33 \cdot 3 \%$ & $25 \%$ & $33.3 \%$ & $16.7 \%$ & $50 \%$ \\
\hline $\begin{array}{l}\text { 6.Interpretación de } \\
\text { textos y diagramas } \\
\text { científicos, resolución } \\
\text { de problemas a partir } \\
\text { de las leyes de los } \\
\text { gases ideales }\end{array}$ & $33.3 \%$ & $25 \%$ & $41.7 \%$ & $16.7 \%$ & $33.3 \%$ & $50 \%$ & $33.3 \%$ & $16.7 \%$ & $50 \%$ & $25 \%$ & $25 \%$ & $50 \%$ \\
\hline
\end{tabular}

Tabla 3 Resultados de instrumento 3. Fuente Elaboración propia.

\section{Discusión}

La competencia interpretativa se divide en las dimensiones gráfica, lecto-escritora y resolutiva, como se dijo anteriormente. A continuación se presenta la discusión de resultados en la evaluación de cada una de estas dimensiones.

\section{Dimensión resolutiva}

Tal como se evidencia en el anexo 2, es posible observar que en la actividad de iniciación (actividad n. ${ }^{\circ}$ ), la cual es intencionada para la presente dimensión; los estudiantes presentan un nivel de interpretación 1 , con relación a las acciones 
propuestas dentro de la tabla de jerarquización por niveles. Este nivel es alcanzado por el $63 \%$ de los estudiantes, allí se puede realizar inferencias como reconocer los reactivos y productos por medio de los vectores presentados en los diagramas de proceso, formulando así reacciones químicas. Dicho esto, acorde a los resultados obtenidos por Cifuentes y Salcedo (2008), es importante que se realicen más actividades que permitían que el estudiante realice análisis a partir de gráficos.

Sin embargo, es importante mencionar que en este punto los estudiantes no se encontraron familiarizados con la actividad a realizar, ya que al presentar la información de manera diferente empleando diagramas de proceso hace que el proceso interpretativo se desarrolle con mayor dificultad y por ende se presente un nivel uno, siendo concordante con la facilidad que el $46 \%$ de los estudiantes dice poder comprender inferencias para llevar el hilo conductor en la realización de las actividades.

Es posible ver la evolución de los procesos interpretativos solamente cuando se llega a las actividades de finalización, ya que si bien en las actividades de desarrollo los estudiantes dan simple solución a ejercicios de lápiz y papel, estas actividades no demandan una mayor interpretación de los cálculos realizados con los enunciados de cada ejercicio, por lo que la intensión en la ejecución de esta actividades era fortalecer las habilidades del grupo para obtener un nivel de interpretación 1 en total. En las actividades 3 y 4 se presentaron porcentajes 85 y $100 \%$ respectivamente, en la resolución de los ejercicios e identificación de reactivos límite y en exceso.

Lo que permite la evolución de la interpretación a un nivel tres es la realización de actividades complementarias; el $87 \%$ de los estudiantes realiza una hipótesis tipo 2, y con base en los ítems de evaluación de la actividad este nivel de hipótesis demandó a los estudiantes hacer una relación de variables propias de las leyes de los gases con las consecuencias ambientales que ocasionan los gases de efecto invernadero, lo que da respuesta a actividades propias de la vida cotidiana y además dan alternativas para solución de dicha problemática.
En la actividad 5 la intención fue desarrollar los niveles interpretativos por lo que al hacer una actividad por medio de preguntas permite la relación de los cálculos estequiométricos realizados con las temáticas vistas en clase. El $73 \%$ de los estudiantes dieron respuesta a preguntas que corresponden a un nivel 3 de interpretación establecieron relaciones entre los cálculos estequiométricos con la ley de la conservación de la masa de acuerdo a lo realizado por Cruz y Ocampo (2012), los procesos de interpretación están determinados por el nivel de conocimiento que presentan los estudiantes en las temáticas tratadas.

\section{Dimensión gráfica}

Los resultados obtenidos en la construcción de diagramas de flujo evidenciaron que el 50\% de la población posee la habilidad de diagramar información recopilada en guías de laboratorio. En otras actividades el $87 \%$ de los estudiantes describe de manera correcta los diferentes procesos de obtención de las sustancias empleadas, evidenciándose un nivel de interpretación 2. Según la dimensión gráfica, el estudiante se encuentra en este nivel cuando explica de manera óptima cómo se relacionan las variables presentes en un gráfico de carácter científico. En comparación con lo realizado por Cifuentes y Salcedo (2008) concluyen que no se llega a niveles altos de análisis debido a la falta implementación de gráficos; en la presente investigación, aunque hubo pocas actividades correspondientes a la dimensión gráfica, se ve una buena aproximación a esta.

\section{Dimensión lecto-escritora}

Según Ramos (2013), el nivel 1 de la dimensión lecto-escritora implica la reorganización de información de textos de carácter científico, además de realizar clasificaciones, bosquejos, resúmenes y síntesis, de acuerdo a los resultados obtenidos en la actividad n. 2 de iniciación; solo el $12 \%$ de los estudiantes realizó un diagrama de tipo 2 mostrando un nivel un poco más avanzado de interpretación, mientras que los estudiantes que se encuentran en el nivel 1 realizan los diagrama de tipo 1, los cuales son una transcripción de lo expuesto en el texto. En los 
resultados en el ítem 1 del instrumento, el $80 \%$ de los estudiantes no ven dificultades a la hora de realizar diagramas; sin embargo, un alto porcentaje no lo realiza de manera correcta.

Dicho lo anterior, en el nivel de interpretación en la dimensión lecto-escritora de los estudiantes se evidenció el mejoramiento de esta, ya que en las actividades de iniciación tenían un nivel 1, que es la comprensión literal y en las actividades de finalización alcanzaron un nivel 2, el cual es de comprensión inferencial, a diferencia de lo realizado por Cifuentes y Salcedo (2008). Estos autores consideran que los niveles de la competencia interpretativa son meramente lingüísticos ya que dentro de los objetivos por nivel pretenden que el estudiante realice acciones como la elaboración de síntesis, explicación de fenómenos guiados por preguntas y emisión de juicios para la interpretación; una vez desarrolladas las actividades no se llega a niveles altos de análisis debido al bajo dominio conceptual por parte de los estudiantes. En ese sentido, los autores ven pertinente la realización de análisis teóricos de tipo cualitativo, como partir de las situaciones problema propuestas para que el estudiante pueda comprender, declarar y explicar acciones que permiten una evolución para la competencia interpretativa.

Acorde con la información recolectada en el instrumento 3 con el que se pretende analizar la relación con la ingeniería y la asimilación de conceptos, en la secuencia didáctica se obtiene el nivel más alto de interpretación en cada una de las dimensiones de la competencia interpretativa, ya que el mayor nivel de estas es la relación con la vida cotidiana. En este caso los estudiantes están relacionando las actividades con la carrera profesional que están cursando, estos resultados concuerdan con lo obtenido en el trabajo realizado por Pinto (2004), en el cual los estudiantes estiman que es conveniente la relación de la química con la vida cotidiana para una mayor motivación hacia el estudio de la asignatura, por lo que contribuyen a una mejor asimilación de los conceptos.

\section{Consideraciones finales}

El diseño y aplicación de la secuencia didáctica permitió evidenciar que es posible la potenciación de habilidades concernientes a la competencia interpretativa. Si bien se presentó una evolución significativa en los procesos de interpretación dentro de la dimensión resolutiva al partir de un nivel 1 hasta lograr un nivel 3, es importante que para el desarrollo de las demás dimensiones se diseñen la misma cantidad de actividades con el fin de que el estudiante pueda ejecutar acciones que le permitan alcanzar el nivel máximo de interpretación, bien sea grafico o de tipo lecto-escritor.

Dado que en los procesos de aprendizaje no solo influye la disposición que presenta el estudiante, es posible evidenciar la aparición de otro conjunto de factores influyentes, tales como el entorno educativo y la observación, esta última como habilidad perceptiva complementaria a la interpretación. La frecuencia con la que estos factores afectan a los estudiantes establecen una relación con el nivel de interpretación presentado según sus dimensiones, que se ve reflejado en el desarrollo de la competencia interpretativa. Esta conclusión va en la misma línea con lo planteado por Rendón y Zapata (2005), donde las habilidades metacognoscitivas necesarias para el aprendizaje consciente, que inicialmente no se encuentran elaboradas en los sujetos, pueden ser aprendidas a través de un programa en dichas habilidades y por tanto se aprenden no solo conocimientos sino procesos.

La importancia de la aplicación de una secuencia didáctica radica tanto en el planteamiento como en el desarrollo de habilidades; las actividades deben ser planteadas según los contenidos de tal manera que motiven a los estudiantes con el fin de obtener mejores resultados a la hora de interpretar. Es por esto que la intención empleada para el desarrollo de la secuencia fue el enfoque con la ingeniera, acorde con lo desarrollado por Luque (2016); la interpretación se ve favorecida cuando se desarrolla alrededor de temas de interés de los estudiantes, si bien en dicha investigación se habla de la consecución de niveles interpretativos, esta competencia es medida por el desarrollo de una simple actividad, por lo que el presente trabajo de investigación presenta una mayor pertinencia ya que maneja actividades que permiten ver si hay evolución en las habilidades interpretativas. 


\section{Referencias}

Cifuentes, A., y Salcedo, L. (2008). Situaciones problema como punto de partida para fomentar el desarrollo de las competencias interpretativas, argumentativas y propositivas en los estudiantes (tesis de maestría). Universidad Pedagógica Nacional, Bogotá, Colombia.

Cruz, D., y Ocampo, D. (2012). Competencia interpretativa y habilidades de pensamiento (tesis de maestría). Universidad Pedagógica Nacional, Bogotá, Colombia.

Gallego, C. (2012). Enseñanza por competencias para un aprendizaje significativo en matemáticas. Obtenido http://www.bdigital.unal.edu. co/5833/1/43153603.2012.pdf

Luque, Y. (2016). Desarrollo de las competencias interpretativas y argumentativas empleando una secuencia didáctica en el contexto de la función alcohol. MaDoQuím, (4), 102-105.

Martínez , J., y Pinto, G. (2010). La Química como Materia Básica de los Grados de Ingeniería. Real sociedad Española de Química, Madrid,España

Pinto, G. (2004). Ejemplos de la vida cotidiana para el aprendizaje de la química: valoración por alumnos universitarios. Anales de la Real Sociedad Española de Química, (2), 2004, 37-43.
Ramos, Z. (2013). La comprensión lectora como una herramienta básica en la enseñanza de las ciencias naturales. (tesis de maestría). Universidad Nacional de Colombia, Bogotá, Colombia.

Rendón, M., y Zapata, P. (2005). Desarrollo de habilidades de pensamiento y enseñanza de las Ciencias. Enseñanza de las ciencias.Bogotá, Colombia.

Toro, J., Reyes, C., \& Martínez, R. (2007). Fundamentación conceptual, área de ciencias naturales. Obtenido de http://paidagogos.co/pdf/fundamentacion_ciencias.pdf

USTA. (2015). Proyecto educativo del programa de ingeniería mecánica. Obtenido de http://facultadingenieriamecanica. usta.edu.co/images/audio/ PEPingmec_2015.pdf

Vargas, V. (2008). Las competencias interpretativas, una oportunidad y un reto para la educación del siglo XXI. Lima PDF. 


\title{
Anexos
}

Anexo 1. Instrumento 1

\author{
UNIVERSIDAD PEDAGÓGICA NACIONAL \\ FACULTAD DE CIENCIA Y TECNOLOGÍA \\ DEPARTAMENTO DE QUÍMICA \\ COMPETENCIA INTERPRETATIVA
}

Los estudiantes de Licenciatura en Química de la Universidad Pedagógica Nacional adelantan un proyecto sobre competencia interpretativa en las clases de química para estudiantes de ingeniería de la universidad Santo Tomás de Aquino. El siguiente cuestionario consta de un cuadro con una serie de preguntas con las que se pretende medir la frecuencia con la que el estudiante realiza actividades interpretativas; se pide contestar con la mayor sinceridad posible, de antemano se agradece la atención prestada.

Nombre: Código:

1. El siguiente cuadro contiene enunciados y afirmaciones relacionados con actividades propias del proceso de interpretación científica donde debe marcar con una $\mathrm{X}$ el grado de frecuencia con el que usted realiza dichas acciones.

\begin{tabular}{|c|c|c|c|}
\hline \multirow[b]{2}{*}{ Acciones } & \multicolumn{3}{|c|}{ Frecuencia } \\
\hline & Siempre & $\begin{array}{l}\text { Algunas } \\
\text { veces }\end{array}$ & Nunca \\
\hline 1. Al observar un fenómeno lo relaciona con situaciones cotidianas. & & & \\
\hline 2. Cuando lee textos científicos elabora mapas mentales o conceptuales. & & & \\
\hline 3. Cuando su maestro hace un discurso o expone un tema entiende todo a cabalidad. & & & \\
\hline 4. Los conceptos de carácter científico le parecen muy abstractos y difíciles de entende & & & \\
\hline 5. Define conceptos o fenómenos con sus propias palabras. & & & \\
\hline 6. La información de carácter científico presentada en textos le parece aburrida & & & \\
\hline $\begin{array}{l}\text { 7. Si se le presentan documentos para realizar prácticas de laboratorio prefiere gran } \\
\text { contenido de gráficos. }\end{array}$ & & & \\
\hline $\begin{array}{l}\text { 8. Observa patrones o similitudes en contextos científicos para generalizar o deducir } \\
\text { principios. }\end{array}$ & & & \\
\hline 9. Tiene interés por los avances científicos en pro de la humanidad. & & & \\
\hline 10. Las clases de química son monótonas y poco interesantes. & & & \\
\hline
\end{tabular}




\title{
Anexo 2. Instrumento 2
}

\author{
UNIVERSIDAD PEDAGÓGICA NACIONAL \\ FACULTAD DE CIENCIA Y TECNOLOGÍA \\ DEPARTAMENTO DE QUÍMICA \\ COMPETENCIA INTERPRETATIVA
}

Los estudiantes de Licenciatura en Química de la Universidad Pedagógica Nacional adelantan un proyecto sobre competencia interpretativa en las clases de química para estudiantes de ingeniería de la universidad Santo Tomás de Aquino. El siguiente cuestionario consta de un cuadro con una serie de preguntas con las que se pretende evaluar el grado de dificultad que presentaron para usted la realización de las diferentes actividades propuestas; se pide contestar con la mayor sinceridad posible, de antemano se agradece la atención prestada.

Nombre: Código:

1. El siguiente cuadro contiene enunciados y afirmaciones relacionados con las actividades que se realizaron a lo largo de la ejecución de la secuencia didáctica para el desarrollo de los diferentes componentes de la competencia interpretativa por esto debe marcar con una X la dificultad que presenta para usted el desarrollo de dichas actividades.

\begin{tabular}{|c|c|c|c|}
\hline \multirow[b]{2}{*}{ Acciones } & \multicolumn{3}{|c|}{ Grado de dificultad } \\
\hline & Fácil & Difícil & $\begin{array}{c}\text { No lo sé } \\
\text { aun }\end{array}$ \\
\hline 1. Realizo cuadros o diagramas interpretativos a partir de la lectura de textos científicos & & & \\
\hline 2. Realiza hipótesis a partir de fenómenos observables & & & \\
\hline 3. Construye diagramas para dar explicación a procesos industriales & & & \\
\hline $\begin{array}{l}\text { 4. Es capaz de realizar inferencias y llevar hilo conductor en la temática cuando no toda } \\
\text { la información está explícita. }\end{array}$ & & & \\
\hline $\begin{array}{l}\text { 5. Diseña diagramas de flujo para entender claramente el paso a paso en la ejecución d } \\
\text { las prácticas de laboratorio }\end{array}$ & & & \\
\hline 6. Resuelve problemas a partir de analogías & & & \\
\hline 7. Obtiene reacciones químicas a partir de diagramas de procesos & & & \\
\hline 8. Reconoce fenómenos tales como la corrosión en relación al fenómeno REDOX & & & \\
\hline 9. Relaciona la temática de estequiometria con la ley de conservación de la masa & & & \\
\hline 10. Identifica reactivos límite y en exceso en la realización de ejercicios de lápiz y papel & & & \\
\hline
\end{tabular}


Anexo 3. Instrumento 3

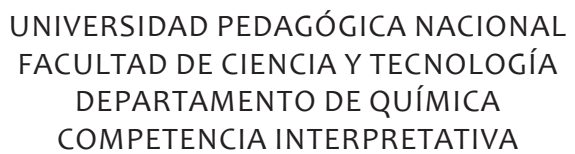

Los estudiantes de Licenciatura en Química de la Universidad Pedagógica Nacional adelantan un proyecto sobre competencia interpretativa en las clases de química para estudiantes de ingeniería de la universidad Santo Tomás de Aquino. el siguiente cuestionario consta de un cuadro con una serie de preguntas con las que se pretende evaluar el progreso de la competencia interpretativa en cada una de sus dimensiones; se pide contestar con la mayor sinceridad posible, de antemano se agradece la atención prestada.

Nombre: Código:

1. El siguiente cuadro contiene enunciados y afirmaciones relacionados con las actividades que se realizaron a lo largo de la ejecución de la secuencia didáctica para el desarrollo de las diferentes dimensiones de la competencia interpretativa, por esto debe marcar su respuesta en cada casilla de acuerdo a su grado en la competencia interpretativa en cada una de las actividades, con una puntuación de 1.0 a 5.0, siendo 5.0 la nota más alta y 1.0 la nota más baja.

\begin{tabular}{|c|c|c|c|c|}
\hline Estrategia metodológica & $\begin{array}{l}\text { Recuerdo la } \\
\text { actividad }\end{array}$ & $\begin{array}{l}\text { Utilidad para } \\
\text { asimilación de } \\
\text { conceptos }\end{array}$ & $\begin{array}{l}\text { Utilidad para } \\
\text { favorecer el } \\
\text { interés }\end{array}$ & $\begin{array}{l}\text { Relación con } \\
\text { la ingeniería }\end{array}$ \\
\hline \multicolumn{5}{|l|}{$\begin{array}{l}\text { 1. Reconocimiento e interpretación de diagramas de } \\
\text { procesos. }\end{array}$} \\
\hline \multicolumn{5}{|l|}{ 2. Interpretación de textos científicos. } \\
\hline \multicolumn{5}{|l|}{$\begin{array}{l}\text { 3. Interpretación de analogías y enunciados para la } \\
\text { resolución de problemas. }\end{array}$} \\
\hline \multicolumn{5}{|l|}{$\begin{array}{l}\text { 4. Interpretación de gráficos para la resolución de } \\
\text { problemas sobre reacciones químicas. }\end{array}$} \\
\hline \multicolumn{5}{|l|}{$\begin{array}{l}\text { 5. Interpretar reacciones químicas, realizar cálculos } \\
\text { estequiométricos a partir de gráficos. }\end{array}$} \\
\hline $\begin{array}{l}\text { 6. Interpretación de diagramas y gráficos con } \\
\text { información de corte científico, resolución de } \\
\text { problemas a partir de las leyes de los gases ideales }\end{array}$ & & & & \\
\hline
\end{tabular}


